\title{
Gridless DOA estimation with finite rate of innovation reconstruction based on symmetric Toeplitz covariance matrix
}

\author{
Tao Chen, Lin Shi and Yongzhi Yu* (D)
}

\section{*Correspondence:}

yuyongzhi@hrbeu.edu.cn

The College of Information and Communication Engineering,

Harbin Engineering University,

Harbin, 150001, China

\section{Springer Open}

\begin{abstract}
Due to the rapid development and wide application of compressed sensing and sparse reconstruction theory, there exists a series of sparsity-based methods for the antenna sensor array direction of arrival (DOA) estimation with excellent performance. However, it is known that this kind of algorithms always suffers from the problem of grid mismatch. To overcome this shortcoming, a gridless DOA estimation algorithm with finite rate of innovation (FRI) based on a symmetric Toeplitz covariance matrix is proposed for uniform linear array (ULA) in this paper. In particular, a multiple measurement vector (MMV) FRI reconstruction model is built by exploiting the covariance data denoised according to covariance fitting criteria rather than the direct data or the original covariance data, which is commonly used in other representative gridless DOA estimation methods. Next, DOA can be retrieved from the recovered covariance matrix by utilizing an annihilating filter because each covariance data is a linear combination of complex exponentials. It guarantees to produce an exact spatial sparse estimate without discretization required by existing sparsity-based DOA estimation methods. Finally, the effectiveness and superiority of the proposed algorithm are demonstrated by numerical simulations.
\end{abstract}

Keywords: Gridless direction of arrival (DOA) estimation, Finite rate of innovation (FRI), Covariance fitting criteria, Annihilating filter

\section{Introduction}

As a typical problem in array signal processing, direction of arrival (DOA) estimation has found wide applications, such as radar, sonar, and wireless communications $[1,2]$. DOA estimation is a process of retrieving the direction parameters of several sources from observed snapshots of receiving antenna sensors. Compared with the traditional subspace-based DOA estimation algorithms represented by MUSIC [3] and ESPRIT [4], the sparsity-based DOA estimation algorithms have more remarkable results in several demanding scenarios, including cases with low signal-to-noise ratio (SNR), limited number of snapshots (even a single snapshot), and highly or completely correlated sources [5-7].

(c) The Author(s). 2020 Open Access This article is licensed under a Creative Commons Attribution 4.0 International License, which permits use, sharing, adaptation, distribution and reproduction in any medium or format, as long as you give appropriate credit to the original author(s) and the source, provide a link to the Creative Commons licence, and indicate if changes were made. The images or other third party material in this article are included in the article's Creative Commons licence, unless indicated otherwise in a credit line to the material. If material is not included in the article's Creative Commons licence and your intended use is not permitted by statutory regulation or exceeds the permitted use, you will need to obtain permission directly from the copyright holder. To view a copy of this licence, visit http://creativecommons.org/licenses/by/4.0/. 
Because of these attractive properties, sparsity-based DOA estimation algorithms have received extensive attention and research in the past decade. According to the relationship between real locations of sources and spatial discrete grids, the existing sparsity-based DOA estimation algorithms can be classified into three categories: on-grid, off-grid, and gridless.

By assuming that the real directions of incident sources coincide with the certain locations in presupposed discrete grids in space, on-grid sparsity-based DOA estimation algorithms represented by $\ell_{1}$-svd method [8] transform DOA estimation into a linear sparse signal reconstruction problem. In particular, since the grid size is greatly larger than the source number, spatial signals can be extended to a sparse vector, which is composed of virtual numerical values from candidate directions on the grid. After that, DOAs of sources, which represent the locations of non-zero elements in the reconstructed signal, can be obtained by spectral peak searching.

However, these algorithms, which are supported by sparse signal reconstruction and compressed sensing theory, rely heavily on an accurate spatial discrete grid. In case of grid mismatch, the performance of these algorithms will be reduced. To address the issue of grid mismatch, two classes of sparsity-based DOA estimation algorithms named offgrid and gridless have recently been proposed. Off-grid methods interpolate the original spatial discrete grid, thus improving the performance of on-grid sparsity-based DOA estimation algorithms $[9,10]$. Gridless algorithms estimate DOAs of sources directly in continuous angle range, which completely avoids the problem of grid mismatch [11, 12].

The existing gridless algorithms can be divided into three main categories: based on the atomic norm minimization (ANM) [13, 14], covariance fitting criterion [15, 16], and finite rate of innovation (FRI) [17], respectively. The atomic norm, which was first proposed by [18], is exploited to deal with the grid mismatch problem by generalizing the common norms for finite dimension vectors, e.g., $\ell_{1}$ norm and nuclear norm, to infinite dimension. However, most of these algorithms have been used in spectral estimation, which can be regarded as DOA estimation in single measurement vector (SMV), and not suitable for DOA estimation in multi-snapshot scenarios.

In the case of multiple snapshots, the sparse and parametric approach (SPA) [19] (a.k.a. the gridless sparse iterative covariance-based estimation method, GLS for short) has been proposed by utilizing the covariance fitting criterion. Firstly, SPA recovers the matrix satisfying symmetric Toeplitz structure from the real covariance data by solving a semidefinite programming (SDP) problem based on minimizing the objective function of covariance fitting criterion. Then, DOAs of sources can be retrieved according to the typical Vandermonde decomposition theory for symmetric positive semidefinite Toeplitz matrices.

A recent proposed algorithm based on FRI signal reconstruction theory has shown its potentials [20-22]. In this algorithm, a reconstruction model of spatial FRI signal is first established taking advantage of the first Bessel functions to approximate the data in the covariance matrix of array received signals. Afterwards, the DOAs of sources can be estimated according to the properties of annihilating filter $[17,23]$ and optimization theory [24]. As the most attractive part of this gridless algorithm, an overcomplete dictionary with infinite dimensions has been established, which can be exploited to represent the relationship between FRI signals in continuous domain and array received signals. However, it does not consider the influence of noise on DOA estimation model. 
This paper aims at developing the performance of gridless DOA estimation algorithms by combining the covariance fitting criteria and FRI signal reconstruction theory. Through a series of reasonable assumptions for far-field, narrowband sources and noises, which are usually appear in the literatures focusing on DOA estimation, the covariance matrix of array received signals satisfying the symmetric Toeplitz structure can be reformulated. After reconstructing the symmetric Toeplitz structure matrix, which can effectively suppress the influence of noise on DOA estimation model, the FRI signal reconstruction theory is used to recover the DOAs of sources directly.

The rest of this article is arranged as follows. Section 2 describes the data model for DOA estimation. Then, a gridless DOA estimation algorithm based on the covariance fitting criteria and FRI signal reconstruction theory is proposed in Section 3. Section 4 presents the results of numerical simulations, and this paper is concluded in Section 5.

The meanings of notations in this paper are explained as follows. Firstly, unbolded letters (e.g., a and A), lower-case bold letters (e.g., a), and upper-case bold letters (e.g., A) are used to represent scalars, vectors, and matrices, respectively. Moreover, $(\cdot)^{T},(\cdot)^{*}$, and $(\cdot)^{H}$ are transpose, conjugate, and conjugate transpose of $(\cdot) . \mathbb{E}\{\cdot\}$ is exploited to represent the expectation of $\cdot . \mathbb{Z}, \mathbb{R}, \mathbb{C}$ is the set of integer, real, and complex numbers, respectively. $\operatorname{tr}\{\cdot\}$ is traces of matrix $\{\cdot\} . \mathbf{A} \succeq 0$ represents that $\mathbf{A}$ is a positive semidefinite matrix, that is to say, its eigenvalues are non-negative. Finally, $j=\sqrt{-1}$ denotes the imaginary unit.

\section{The data model for DOA estimation}

Considering the existence of $K \in \mathbb{Z}_{+}$far-field narrowband sources in space, they impinge on an ULA with $M \in \mathbb{Z}_{+}$identical antenna sensors from directions $\boldsymbol{\theta}=\left[\theta_{1}, \theta_{2}, \ldots, \theta_{K}\right]$, respectively. In this paper, $\theta_{k}(k=1, \ldots, K)$ is defined as the angle between direction of $k$ th source and normal of array. Meanwhile, we only concern with the one-dimensional angular space, implying that $\theta_{k} \in[-\pi / 2, \pi / 2]$.

When the array element spacing is $\lambda / 2$, where $\lambda$ is the wavelength of the incident source, and the coordinate origin is set as the phase reference point, the received signal model of the antenna array can be expressed as follows:

$$
\mathbf{Y}=\mathbf{A}(\boldsymbol{\theta}) \mathbf{S}+\mathbf{E},
$$

where $\mathbf{Y}=[\mathbf{y}(1), \ldots, \mathbf{y}(L)] \in \mathbb{C}^{M \times L}$ and $\mathbf{S} \in \mathbb{C}^{K \times L}$ and $\mathbf{E} \in \mathbb{C}^{M \times L}$ are both similarly defined, representing the matrix of sources and noise, respectively. $L \in \mathbb{Z}_{+}$is the number of snapshots. In addition, the form of array manifold matrix is $\mathbf{A}(\boldsymbol{\theta})=\left[\mathbf{a}\left(\theta_{1}\right), \ldots, \mathbf{a}\left(\theta_{K}\right)\right] \in$ $\mathbb{C}^{M \times K}$, where $\mathbf{a}\left(\theta_{k}\right)=\left[a_{1}\left(\theta_{k}\right), \ldots, a_{M}\left(\theta_{k}\right)\right]^{T} \in \mathbb{C}^{M}$ is the steering vector of the $k$ th source.

The received signals at two different antenna sensors differ by a phase shift, which can be expressed as the relative distance of the antennas and the locations of point sources in the angular space. Hence, the specific form of $a_{m}\left(\theta_{k}\right)$ can be expressed as:

$$
a_{m}\left(\theta_{k}\right)=e^{-j \pi(m-1) \sin \left(\theta_{k}\right)}, m=1,2, \ldots, M, k=1,2, \ldots, K .
$$

We assume that the noise $\mathbf{e}(t)(t=1, \ldots, L)$ are complex Gaussian white noise and not correlated with the sources. Meanwhile, the sources are assumed to be independent of each other. Under the assumptions above, the covariance matrix of received signals is: 


$$
\begin{aligned}
\mathbf{R} & =\mathbb{E}\left\{\mathbf{Y} \mathbf{Y}^{H}\right\}=\mathbf{A} \mathbb{E}\left\{\mathbf{S S}^{H}\right\} \mathbf{A}^{H}+\mathbb{E}\left\{\mathbf{E} \mathbf{E}^{H}\right\} \\
& =\operatorname{Adiag}\{\mathbf{p}\} \mathbf{A}^{H}+\sigma \mathbf{I} \\
& =\sum_{k=1}^{K} p_{k} \mathbf{a}\left(\theta_{k}\right) \mathbf{a}^{H}\left(\theta_{k}\right)+\sigma \mathbf{I},
\end{aligned}
$$

where $\operatorname{diag}\{\mathbf{p}\}$ is a diagonal matrix formed by vector $\mathbf{p}=\left[p_{1}, \ldots, p_{K}\right]^{T} \in \mathbb{R}_{+}^{K}$, which denote the power parameters of sources. $\sigma \in \mathbb{R}_{+}$denotes the power parameter of noise, and $\mathbf{I}$ is the $M$ dimensional unit matrix.

Substituting formula (2) into formula (3), we can obtain the data of the $\beta$ th column in $\alpha$ th row of the covariance matrix which is:

$$
\mathbf{R}(\alpha, \beta)=\sum_{k=1}^{K} p_{k} e^{j \pi(\beta-\alpha) \sin \left(\theta_{k}\right)}+\sigma \delta_{\alpha \beta}, \alpha, \beta=1, . ., M,
$$

where $\delta_{\alpha \beta}=1$ when $\alpha=\beta$ or $\delta_{\alpha \beta}=0$ otherwise.

Obviously, the covariance matrix of received signals is a symmetric Toeplitz matrix which is determined by $M$ parameters and can be expressed as:

$$
\mathbf{R}=\mathbf{T}(\mathbf{u})+\sigma \mathbf{I}=\left[\begin{array}{llll}
u_{1} & u_{2} & \cdots & u_{M} \\
u_{2}^{*} & u_{1} & \cdots & u_{M-1} \\
\vdots & \vdots & \ddots & \vdots \\
u_{M}^{*} & u_{M-1}^{*} & \cdots & u_{1}
\end{array}\right]+\sigma \mathbf{I},
$$

where $\mathbf{T}(\mathbf{u})$ is a symmetric Toeplitz matrix with the first row $\mathbf{u}=\left[u_{1}, \ldots, u_{M}\right], u_{1} \in \mathbb{R}_{+}$ and $u_{2}, \ldots, u_{M} \in \mathbb{C}$. The specific form of $u_{m}$ can be written as:

$$
u_{m}=\sum_{k=1}^{K} p_{k}\left(e^{j \pi \sin \left(\theta_{k}\right)}\right)^{m-1}, m=1,2, \ldots, M .
$$

It is worth noting that the covariance matrix of the array received signals satisfies the above mathematical model only under the premise of ensuring the sources uncorrelated and Gaussian white noise. In other words, the algorithm proposed in this paper is not suitable for correlated sources or other types of noise.

Up to now, we have successfully transformed the DOA estimation problem into the estimation of parameters $p_{k}, \theta_{k}$, and $\sigma$. In this paper, because only the DOAs of sources are concerned, the power parameters of sources $p_{k}$ and noise $\sigma$ can be ignored. Next, the method of estimating parameters $u_{m}$ by covariance fitting criterion and parameters $\theta_{k}$ by FRI signal reconstruction theory will be discussed, respectively.

\section{Methods}

Actually, due to the limitation of snapshot number, the covariance matrix of the received signals can hardly satisfy the form shown in formula (5), even if the sources and noise satisfy the assumed conditions. In practical applications, the covariance matrix is estimated with $L$ snapshots as follows:

$$
\hat{\mathbf{R}}=\mathbb{E}\left\{\mathbf{Y} \mathbf{Y}^{H}\right\}=\frac{1}{L} \sum_{l=1}^{L} \mathbf{y}(l) \mathbf{y}^{H}(l)
$$


Therefore, our first task is to recover the matrix satisfying symmetric Toeplitz structure from the real covariance data $\hat{\mathbf{R}}$ by solving a SDP problem based on minimizing the objective function of covariance fitting criterion.

\subsection{The symmetric Toeplitz structure covariance matrix reconstruction}

When $\hat{\mathbf{R}}$ and $\mathbf{R}$ are both invertible, the objective function of parameter estimation based on covariance fitting criterion is $[15,16]$ :

$$
f(\boldsymbol{\theta}, \mathbf{p}, \sigma)=\left\|\mathbf{R}^{-\frac{1}{2}}(\hat{\mathbf{R}}-\mathbf{R}) \hat{\mathbf{R}}^{-\frac{1}{2}}\right\|_{F}^{2}, \boldsymbol{\theta} \in \mathbb{R}^{K}, \theta_{k} \in\left[-\frac{\pi}{2}, \frac{\pi}{2}\right], \mathbf{p} \in \mathbb{R}_{+}^{K}, \sigma \in \mathbb{R}_{+} .
$$

From the previous analysis, we can see that when the parameters $\boldsymbol{\theta}, \mathbf{p}$, and $\sigma$ satisfy their respective constraints, $\mathbf{R}$ is a symmetric Toeplitz matrix composed of parameter $\mathbf{u}$, and $\mathbf{R}^{-1}$ exists in the presence of noise. Then, $\hat{\mathbf{R}}^{-1}$ exists with probability one if $M \leqslant L$. When $L<M$, which rarely happens, the covariance fitting criterion and the recovery process of parameter $\mathbf{u}$ are basically similar, so this situation will be not discussed in this paper.

Formula (8) can be rewritten as:

$$
\begin{aligned}
f(\boldsymbol{\theta}, \mathbf{p}, \sigma) & =\operatorname{tr}\left\{\mathbf{R}^{-\frac{1}{2}}(\hat{\mathbf{R}}-\mathbf{R}) \hat{\mathbf{R}}^{-1}(\hat{\mathbf{R}}-\mathbf{R}) \mathbf{R}^{-\frac{1}{2}}\right\} \\
& =\operatorname{tr}\left\{\left(\mathbf{R}^{-1} \hat{\mathbf{R}}-\mathbf{I}\right)\left(\mathbf{I}-\hat{\mathbf{R}}^{-1} \mathbf{R}\right)\right\} \\
& =\operatorname{tr}\left\{\mathbf{R}^{-1} \hat{\mathbf{R}}\right\}+\operatorname{tr}\left\{\hat{\mathbf{R}}^{-1} \mathbf{R}\right\}-2 M .
\end{aligned}
$$

According to the description of $\mathbf{R}$ in formula (5), the minimization of $f(\boldsymbol{\theta}, \mathbf{p}, \sigma)$ is equivalent to:

$$
\begin{aligned}
& \min _{\boldsymbol{\theta}, \mathbf{p}, \sigma} f(\boldsymbol{\theta}, \mathbf{p}, \sigma), \text { s.t. } \mathbf{p} \in \mathbb{R}_{+}^{K}, \sigma>0 \\
\Leftrightarrow & \min _{\mathbf{u}, \sigma>0} \operatorname{tr}\left\{\mathbf{R}^{-1} \hat{\mathbf{R}}\right\}+\operatorname{tr}\left\{\hat{\mathbf{R}}^{-1} \mathbf{R}\right\}, \text { s.t. } \mathbf{R}=\mathbf{T}(\mathbf{u})+\sigma \mathbf{I}, \mathbf{T}(\mathbf{u}) \succeq 0 \\
\Leftrightarrow & \min _{\mathbf{u}, \sigma>0} \operatorname{tr}\left\{\hat{\mathbf{R}}^{\frac{1}{2}} \mathbf{R}^{-1} \hat{\mathbf{R}}^{\frac{1}{2}}\right\}+\operatorname{tr}\left\{\hat{\mathbf{R}}^{-1} \mathbf{R}\right\}, \text { s.t. } \mathbf{R}=\mathbf{T}(\mathbf{u})+\sigma \mathbf{I}, \mathbf{T}(\mathbf{u}) \succeq 0 .
\end{aligned}
$$

In formula (10), the equivalence between the first two optimization problems is obtained according to the Vandermonde decomposition theorem of PSD Toeplitz matrix [12], that is, any PSD Toeplitz matrix $\mathbf{T}(\mathbf{u})$ admits the Vandermonde decomposition:

$$
\mathbf{T}(\mathbf{u})=\mathbf{A}(\boldsymbol{\theta}) \operatorname{diag}(\mathbf{p}) \mathbf{A}^{H}(\boldsymbol{\theta}),
$$

where $p_{k}>0$ and $\theta_{k}, k=1, \ldots, K$ are distinct.

Suppose there is a matrix $\mathbf{X} \in \mathbb{C}^{M \times M}$, which satisfies:

$$
\mathbf{X}-\hat{\mathbf{R}}^{\frac{1}{2}} \mathbf{R}^{-1} \hat{\mathbf{R}}^{\frac{1}{2}} \succeq 0,
$$

so that formula (10) can be further simplified to:

$$
\begin{aligned}
(10) \Leftrightarrow & \min _{\mathbf{X}, \mathbf{u}, \sigma>0} \operatorname{tr}(\mathbf{X})+\operatorname{tr}\left\{\hat{\mathbf{R}}^{-1} \mathbf{R}\right\}, \\
& \text { s.t. } \mathbf{R}=\mathbf{T}(\mathbf{u})+\sigma \mathbf{I}, \mathbf{T}(\mathbf{u}) \succeq 0 \text { and } \mathbf{X}-\hat{\mathbf{R}}^{\frac{1}{2}} \mathbf{R}^{-1} \hat{\mathbf{R}}^{\frac{1}{2}} \succeq 0 \\
\Leftrightarrow & \min _{\mathbf{X}, \mathbf{u}, \sigma>0} \operatorname{tr}(\mathbf{X})+\operatorname{tr}\left\{\hat{\mathbf{R}}^{-1} \mathbf{R}\right\}, \\
& \text { s.t. } \mathbf{R}=\mathbf{T}(\mathbf{u})+\sigma \mathbf{I},\left[\begin{array}{lll}
\mathbf{X} & \hat{\mathbf{R}}^{\frac{1}{2}} & \\
\hat{\mathbf{R}}^{\frac{1}{2}} & \mathbf{R} & \\
& \mathbf{T}(\mathbf{u})
\end{array}\right] \succeq 0 .
\end{aligned}
$$


The simplified SDP formulation as shown in formula (13) can be addressed by any optimization operator, e.g., SDPT or SeDuMi, which are included in CVX toolbox [25]. The parameter $\mathbf{u}$ can be obtained by solving the SDP formulation. In other existing gridless DOA estimation algorithms based on covariance fitting criteria, the DOAs of sources will be retrieved after reconstructing $\mathbf{T}(\mathbf{u})$ exploiting subspace-based methods, such as MUSIC and ESPRIT, or the classical Vandermonde decomposition theory for positive semidefinite Toeplitz matrices, such as SPA. Because of the high complexity of subspace algorithms, which involve peak search or more than one eigenvalue decomposition process, SPA is obviously more efficient and practical.

After the matrix $\mathbf{T}(\mathbf{u})$ satisfying the symmetric Toeplitz structure is obtained, a postprocessing process is introduced to ensure that the decomposition of $\mathbf{T}(\mathbf{u})$ is unique in SPA. The specific method is as follows:

$$
\begin{aligned}
\mathbf{T}(\hat{\mathbf{u}}) & =\mathbf{T}\left(\mathbf{u}^{*}\right)-\lambda_{\min }\left\{\mathbf{T}\left(\mathbf{u}^{*}\right)\right\} \mathbf{I}, \\
\hat{\sigma} & =\sigma^{*}+\lambda_{\min }\left\{\mathbf{T}\left(\mathbf{u}^{*}\right)\right\},
\end{aligned}
$$

where $\left(\mathbf{u}^{*}, \sigma^{*}\right)$ is the solution of the SDP and $(\hat{\mathbf{u}}, \hat{\sigma})$ is the result of postprocessing. $\lambda_{\min }\left\{\mathbf{T}\left(\mathbf{u}^{*}\right)\right\}$ denotes the minimum eigenvalue of $\mathbf{T}\left(\mathbf{u}^{*}\right)$.

The postprocessing of SPA essentially separates the signal parameters $\boldsymbol{\theta}, \mathbf{p}$ and the noise parameters $\sigma$ in the estimated covariance matrix to ensure the accuracy of the estimation results. However, in this paper, only the parameter $\boldsymbol{\theta}$ is concerned, rather than the estimation accuracy of the parameters $\mathbf{p}$ and $\sigma$. According to the characteristic description of the covariance matrix optimized by the covariance fitting criterion, it is not difficult to find that the off-diagonal elements of $\mathbf{T}(\mathbf{u})$ are all weighted sum of uniformly sampled complex exponentials when $\pi \sin \left(\theta_{k}\right)$ is considered as a new set of parameters. Such a set of parameters can be uniquely reconstructed from the observation vector composed of the off-diagonal elements of $\mathbf{T}(\mathbf{u})$ by using FRI signal reconstruction theory [23].

\subsection{DOA estimation by FRI signal reconstruction theory}

As one of the main differences with SPA, the algorithm proposed in this paper does not need to deal with the results of SDP as shown in formula (13). That is to say, the parameter $\mathbf{u}^{*}$ is the same as $\hat{\mathbf{u}}$. In order to express conveniently, the symbol $\mathbf{u}$ is used to replace the two parameters mentioned above.

Since parameter $\mathbf{u}$ is completely determined by $2 K$ parameters, i.e., $\theta_{k}(k=1, \ldots, K)$ and $p_{k}(k=1, \ldots, K)$, so it is a FRI signal with the rate of innovation:

$$
\rho=\frac{2 K}{\pi}
$$

As the key of FRI signal reconstruction theory, the next task is to determine the coefficients of annihilation filter equations satisfied by parameter $\mathbf{u}$. We can find that the parameters $u_{m}(m=2, \ldots, M)$ are all a collection of sums of $K$ complex exponential functions. It is worth noting that the noise parameter $\sigma$ only affects the diagonal elements in the covariance matrix, i.e., $u_{1}$, under the assumption of Gaussian white noise. Therefore, in order to suppress the influence of Gaussian white noise, we only use the off-diagonal elements in $\mathbf{T}(\mathbf{u})$ to construct the annihilation equation. 
Then, suppose there is filter as follows:

$$
C(z)=\prod_{k=1}^{K}\left(1-g_{k} z^{-1}\right)=\sum_{n=0}^{K} c_{n} z^{-n},
$$

where $C(z)$ has $K$ zeros at $g_{k}=e^{j \pi \sin \left(\theta_{k}\right)}(k=1, \ldots, K)$, and $c_{n}$ is the coefficients of filter $C(z)$. So that we can have:

$$
\begin{aligned}
\sum_{n=0}^{K} c_{n} u_{m-n} & =\sum_{n=0}^{K} c_{n} \sum_{k=1}^{K} p_{k} g_{k}^{m-n-1} \\
& =\sum_{k=1}^{K} p_{k}\left(\sum_{n=0}^{K} c_{n} g_{k}^{-n}\right) g_{k}^{m-1} \\
& =\sum_{k=1}^{K} p_{k}\left(C\left(g_{k}\right)\right) g_{k}^{m-1}=0 .
\end{aligned}
$$

Thus, the parameters $u_{m}=\sum_{k=1}^{K} p_{k} g_{k}^{m-1}(m=2, \ldots, M)$ are annihilated by the filter $C(z)$, which can be called as annihilated filter.

In matrix form, the system in formula (17) is equivalent to:

$$
\left[\begin{array}{llll}
u_{K+2} & u_{K+1} & \cdots & u_{2} \\
u_{K+3} & u_{K+2} & \cdots & u_{3} \\
\vdots & \vdots & \ddots & \vdots \\
u_{M} & u_{M-1} & \cdots & u_{M-K}
\end{array}\right]\left[\begin{array}{l}
c_{0} \\
c_{1} \\
\vdots \\
c_{K}
\end{array}\right]=\mathbf{0} .
$$

Owing to $M-1$ values $u_{m}$ are available and there are $K+1$ unknown filter coefficients, we need at least $K+1$ equations, and therefore, $M$ must be greater or equal to $2 K+2$, i.e., $M \geq 2 K+2$. By solving the linear equations in the form of formula (18), we can get the coefficients of annihilated filter $c_{k}(k=0,1, \ldots, K)$.

At last, once the filter coefficients $c_{k}$ are found, the values $g_{k}(k=1, \ldots, K)$ are simply the roots of the annihilating filter and the DOAs of sources $\theta_{k}$ can be estimated by:

$$
\sin \left(\hat{\theta_{k}}\right)=-\arg \left[\operatorname{roots}\left(\sum_{n=0}^{K} c_{n} z^{-n}\right)\right] / \pi .
$$

where roots (.) is the operator of finding the zero points of polynomial (.) and $\arg (\cdot)$ denotes the phase corresponding to complex (.).

The process of proposed algorithm for DOA estimation is presented in Table 1. The covariance matrix $\mathbf{R}$ is firstly restructured by solving an SDP formulation. Then, the result of DOA estimation can be obtained by solving a linear equation and finding roots of polynomial. Finally, we can see that the proposed algorithm only takes advantage of the covariance data of received signals, which does not depend on the spatial discrete grid, so the proposed algorithm is a gridless DOA estimation algorithm.

\subsection{Some discussions}

In this section, we will analyze the differences and connections between the proposed algorithm and the existing advanced algorithm, so as to more clearly illustrate the innovation of this paper. 
Table 1 The process of proposed algorithm

\begin{tabular}{ll}
\hline Input & The number of sources $K$, antenna sensors $M$ and snapshots $L$, the received signal matrix $\mathbf{Y}$ \\
Output & The DOA estimation results $\hat{\theta}_{k}(k=1, \ldots, K)$ \\
\hline Step 1 & Calculate the covariance matrix of received signals $\hat{\mathbf{R}}$ by $(7)$ \\
Step 2 & Estimate the parameters $u_{m}$ in the solution of the SDP as shown in (13) \\
Step 3 & $\begin{array}{l}\text { Acquire the coefficients of annihilated filter } c_{k} \text { according to the linear equations in the form of } \\
\text { formula (18) }\end{array}$ \\
Step 4 & Find the roots of polynomial $g_{k}$ with coefficients $c_{k}$ \\
Step 5 & Determine the final DOA estimation results by solving (19) \\
\hline
\end{tabular}

Firstly, the algorithm proposed in this paper is closely related to SPA in reference [19]. Both algorithms adopt the same covariance fitting criteria to recover the covariance matrix satisfying the symmetric Toeplitz structure, which not only reduces the influence of limited snapshot number on the statistical data, but also suppresses the Gaussian white noise. However, due to the difference of the later estimation methods of parameters $\theta_{k}$, the performance of the two algorithms is different.

\subsubsection{Connection to SPA}

There are two main differences between the proposed algorithm and SPA. One is that SPA makes further processing on the optimization results of SDP, i.e., $\mathbf{u}^{*}$ and $\sigma^{*}$, while the proposed algorithm uses the above results directly.

SPA separates the estimation of signal parameters $\boldsymbol{\theta}, \mathbf{p}$ and noise parameter $\sigma$ through postprocessing. However, the proposed algorithm does not care about the noise parameter $\sigma$, so it directly uses the off-diagonal elements in $\mathbf{T}(\mathbf{u})$ to recover the DOA parameter $\boldsymbol{\theta}$ of the incident sources. This can not only achieve the purpose of Gaussian white noise suppression, but also reduce the complexity of the algorithm, which will be analyzed in detail later.

The other difference is the method of obtaining the parameter $\boldsymbol{\theta}$. SPA performs the Cholesky decomposition on the positive semidefinite matrix $\mathbf{T}(\mathbf{u})$ satisfying the symmetric Toeplitz structure. Then, the result of decomposition is utilized to construct a pair of matrix pencil. The final estimation result of the parameter $\boldsymbol{\theta}$ is included in the generalized eigenvalues of the matrix pencil. As described above, the proposed algorithm is different from SPA, which does not involve multiple complex matrix decomposition operations, but only needs to find the solution of a system of linear equations with $K+1$ dimension. This can obviously further improve the efficiency of the algorithm.

Of course, the cost of these improvements is the reduction of freedom. At the same time, the proposed algorithm cannot guarantee the estimation accuracy of noise parameter $\sigma$. However, neither of them is the main content of this paper.

\subsubsection{Connection to FRIDA}

Next, as a successful application of FRI signal reconstruction theory in DOA estimation, the FRI sampling-based algorithm for DOA finding (FRIDA) proposed in [21] has received a lot of attention and research. Strictly speaking, the proposed algorithm in this paper can be considered as a simplified version of FRIDA in special scenarios. Obviously, this special scenario refers to ULA. It is a simplification of FRIDA that no linear mapping matrix between the unknown uniformly sampled sinusoidal signals and the known 
non-uniform measurements is needed. Therefore, there are two differences between the proposed algorithm and FRIDA.

On the one hand, FRIDA uses the Bessel function of the first kind to establish a linear mapping from the Fourier series coefficients of unknown sources, which can be regarded as the uniformly sampled sinusoids, to the given array receiving signals. However, the proposed algorithm does not include this process. Because of the particularity of ULA, the covariance matrix of the received signals can be directly expressed in the form of weighted sum of uniformly sampled complex exponentials, instead of constructing redundant mapping matrix. In this way, the complexity of the algorithm is greatly reduced, but the proposed algorithm is no longer suitable for other array geometry, such as SLA and planar array.

On the other hand, FRIDA takes the raw data of the array received signals or the off-diagonal elements in the covariance matrix calculated from the raw data as the measurements. In contrast, the covariance data of array received signals optimized by covariance fitting criterion is adopted to estimate the DOAs of sources in the proposed algorithm. This method can not only eliminate the influence of the limited number of snapshots on the statistical data, but also suppress the Gaussian white noise such that it can improve the estimation accuracy.

\subsubsection{Computational complexity}

At last, in order to more clearly show the differences with other methods in the computational complexity, specific analysis is given in this section.

In MUSIC algorithm [3], the parts that play a leading role in computing complexity include the calculation of covariance matrix, its eigenvalue decomposition, and the process of peak searching. Their corresponding computational complexity is as follows: $O\left(M^{2} L\right), O\left(M^{3}\right)$, and $O\left(M^{2} N\right)$, where $N$ is the number of grid points. According to the analysis of reference [8], the complexity of $\ell_{1}$-svd algorithm is $O\left(K^{3} N^{3}\right)$. The OGSBL algorithm proposed in [9] mainly includes SVD decomposition with complexity $O\left(\max \left(M L^{2}, M^{2} L\right)\right)$ and iteration having a computational complexity of order $O\left(M N^{2} T\right)$, where $T$ is number of iterations.

It can be seen that the complexity of the above three algorithms is closely related to the number of spatial discrete grid points $N$. Generally, because $N \gg M$, the parameter $N$ usually determines the computational complexity of the above three algorithms.

Reference [19] gives the calculation method of the computational complexity of SPA. The computation of $\hat{\mathbf{R}}^{\frac{1}{2}}$ takes $O\left(M^{2} L+M^{3}\right)$ flops. The postprocessing takes $O\left(M^{3}\right)$ flops. Then, the computational complexity of SDP is $O\left(M^{6.5}\right)$. At last, the Vandermonde decomposition of matrix takes $O\left(M^{2}\right)$ flops.

In the proposed algorithm, the complexity of $\hat{\mathbf{R}}^{\frac{1}{2}}$ calculation and SDP process is the same as SPA. In addition, the complexity of the proposed algorithm should also include a process of solving $K+1$ dimensional linear equations, which takes $O\left((K+1)^{3}\right)$ flops. Due to the condition $M \geq 2 K+2$, the complexity of the proposed algorithm is obviously less than that of SPA. This conclusion will be further confirmed in the simulation experiment of this paper.

The computational complexity of the five DOA estimation algorithms involved in this paper is recorded in Table 2. 
Table 2 The computational complexity of the five DOA estimation algorithms

\begin{tabular}{ll}
\hline Algorithm & Complexity \\
\hline MUSIC & $O\left(M^{2} L+M^{3}+M^{2} N\right)$ \\
$\ell_{1}$-Svd & $O\left(K^{3} N^{3}\right)$ \\
OGSBL & $O\left(\max \left(M L^{2}, M^{2} L\right)+M N^{2} T\right)$ \\
SPA (except SDP) & $O\left(M^{2} L+M^{3}\right)$ \\
Proposed (except SDP) & $O\left(M^{2} L+(K+1)^{3}\right)$ \\
\hline
\end{tabular}

\section{Experiment}

In this section, we illustrate the performance of the proposed method and compare it with existing DOA estimation algorithms via numerical simulations. The algorithms that we consider include MUSIC [3], $\ell_{1}$-svd [8], OGSBL [9], and SPA [19] corresponding to subspace-based, on-grid, off-grid, and gridless sparsity-based DOA estimation algorithm, respectively. In addition to SPA and the proposed algorithm, all other algorithms need spatial discrete grids with uniform spacing $1^{\circ}$ in the following experiments, implying that the number of discrete grid points is $N=180$. The number of iterations in OGSBL algorithm is $T=1000$. The regularization parameter in $\ell_{1}$-svd algorithm is 2 . In the following, we present four sets of experiments to verify the performance of proposed algorithm.

\subsection{Spectra comparisons}

Considering $K=4$ incident sources with DOAs randomly selected from $\left[-60^{\circ}, 60^{\circ}\right]$ and adopting $M=10$ uniform linear array with element spacing $\lambda / 2$, we compare the spectra and DOA estimation results of aforementioned methods in this subsection. The number of snapshots $L$ is 200 . SNR is $0 \mathrm{~dB}$ and $10 \mathrm{~dB}$ corresponding to Figs. 1 and 2, respectively.

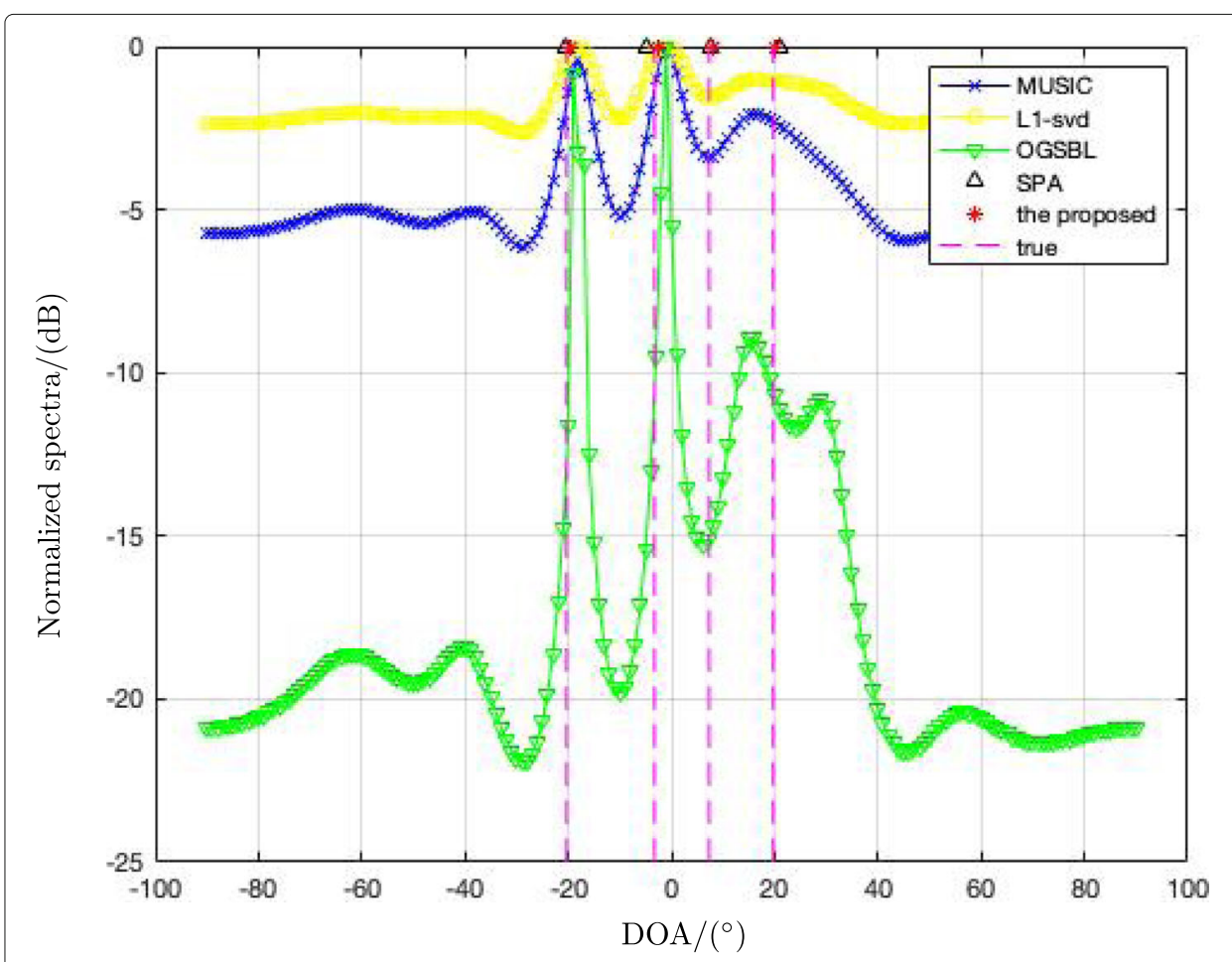

Fig. 1 Spectral of five algorithms in $\mathrm{SNR}=0 \mathrm{~dB}$ 


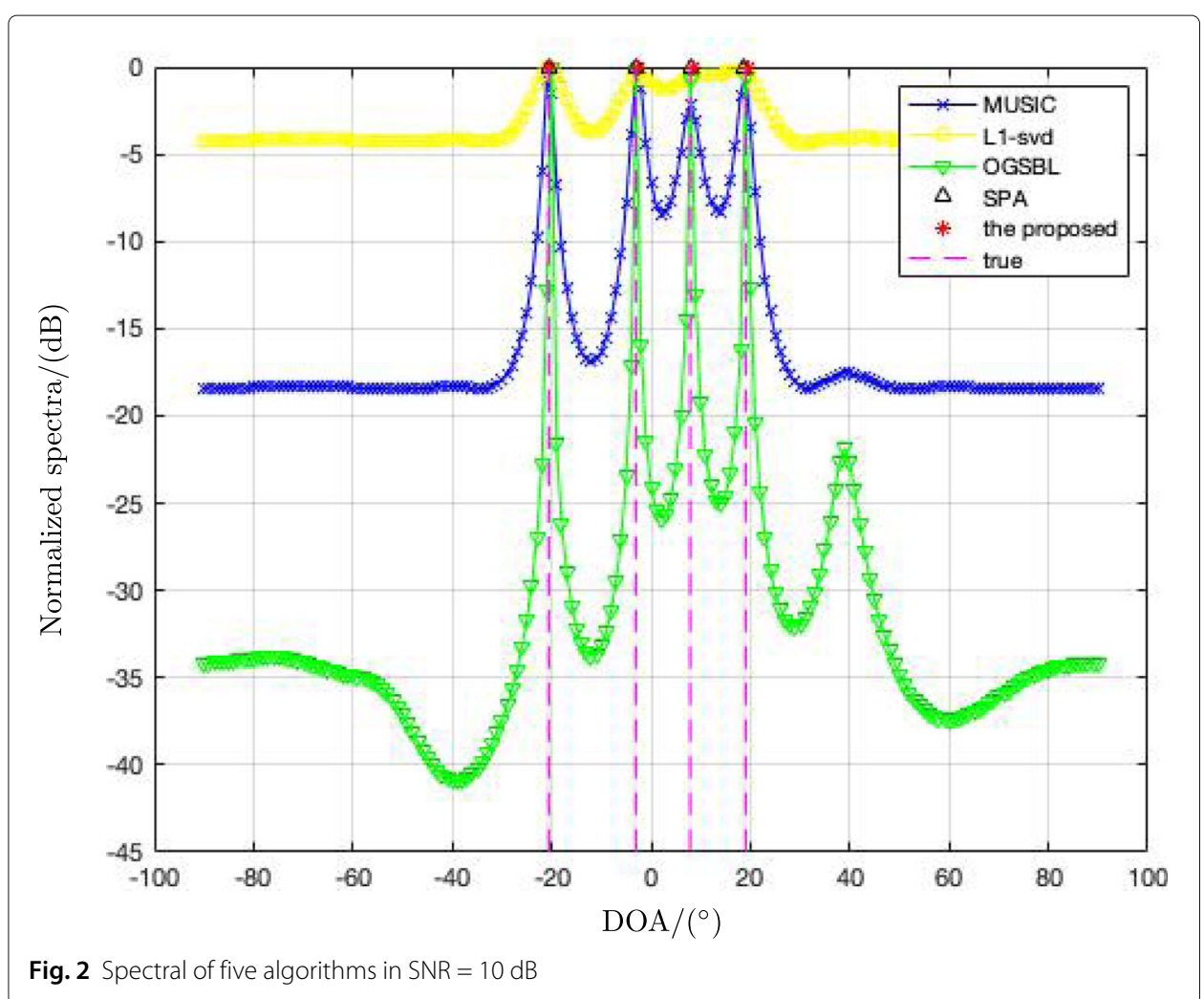

It is not difficult to see that the spectral peaks of all the algorithms are sharper and the estimated results are closer to the true values with the increase of SNR. Meanwhile, the random DOAs of the sources do not fall entirely on the presupposed spatial discrete grids. In the case of low SNR or high SNR, the estimation results of the proposed algorithm are always the closest to the true DOAs of the sources. Due to the limitation of search accuracy, the MUSIC algorithm always deviates from the true source location. The problem of grid mismatch has the greatest impact on the $\ell_{1}$-svd algorithm, sometimes even directly leading to the algorithm failure. Compared with $\ell_{1}$-svd algorithm, OGSBL algorithm has been improved to a certain extent, but still cannot achieve the desired results. Only the SPA and the algorithm proposed in this paper are less affected by the grid mismatch problem.

\subsection{Estimation accuracy comparisons}

In this experiment, the impact of SNR and snapshots on the performance of the algorithm mentioned earlier will be verified. In Fig. 3, the number of sources, antenna sensors, and snapshots is $K=2, M=6$, and $L=100$, respectively. SNR is uniformly transformed from 0 to $20 \mathrm{~dB}$ in step $2 \mathrm{~dB}$. The true incident directions of the sources are randomly selected in $\left[-60^{\circ}, 60^{\circ}\right]$, and the spacing is guaranteed to be greater than $5^{\circ}$. The experimental conditions of Fig. 4 are basically the same as those of Fig. 3, except that SNR is fixed at $10 \mathrm{~dB}$. At the same time, the number of snapshots uniformly changes from 50 to 500 with step 50. One hundred independent Monte Carlo simulations are carried out for both Figs. 3 and 4. The performances of above algorithms are compared according to the values of root mean square error (RMSE), which is defined as: 


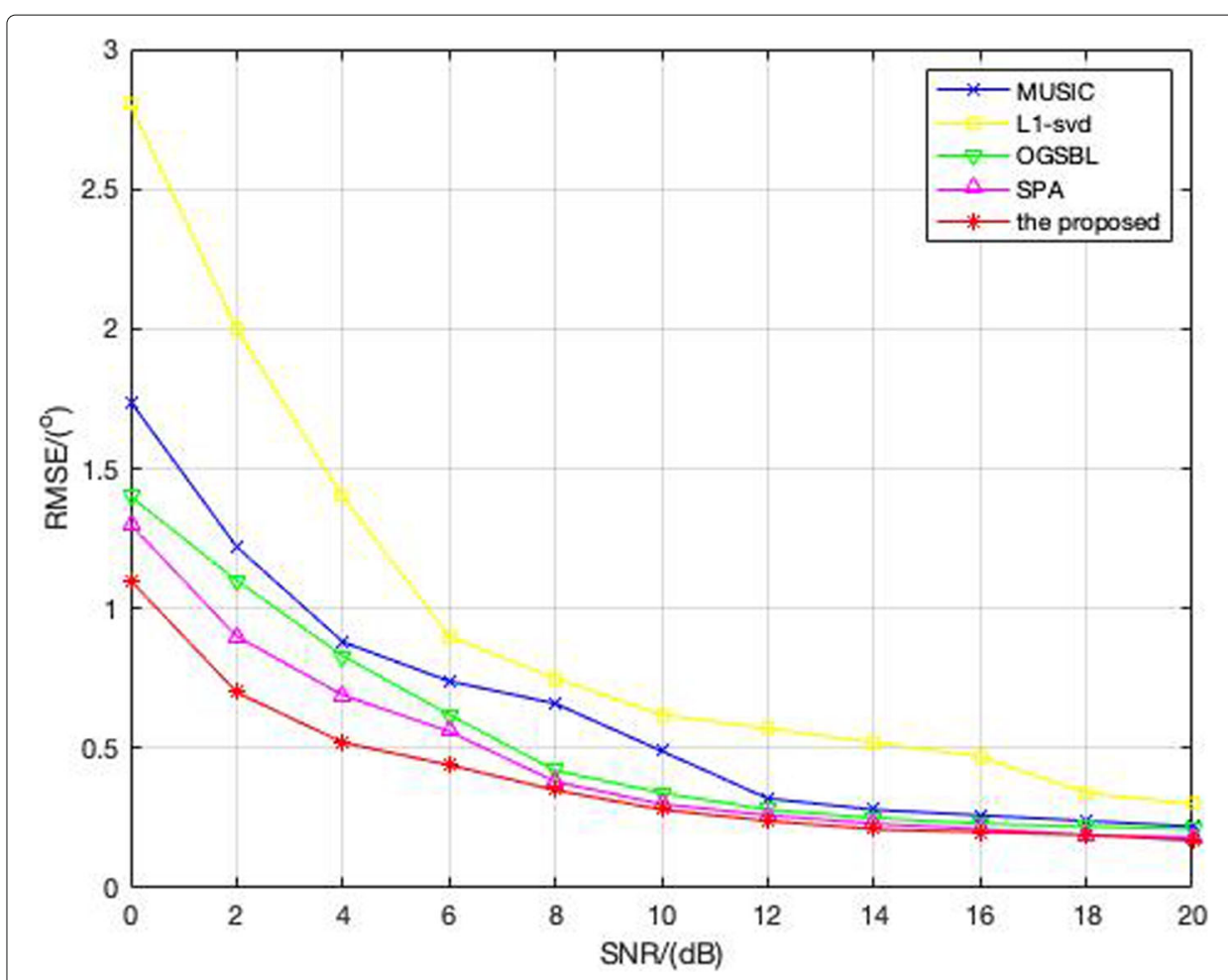

Fig. 3 RMSEs of five algorithms versus SNR

$$
\operatorname{RMSE}=\frac{1}{Q K} \sqrt{\sum_{q=1}^{Q} \sum_{k=1}^{K}\left(\widehat{\theta}_{q, k}-\theta_{k}\right)^{2}},
$$

where $\widehat{\theta}_{q, k}$ denotes the $q$ th estimated value of source incidence angle $\theta_{k}$, and $Q$ is the number of Monte Carlo experiments in this paper.

Figure 3 plots RMSEs of five algorithms under different SNRs. The RMSEs of five algorithms improve constantly with the SNR. Under the same SNR, the proposed algorithm obviously has better estimation performance. The same experimental conclusion can be obtained through Fig. 4, which presents the experimental results under different snapshots.

\subsection{Angle resolution comparisons}

Next, the influence of angle interval between two sources, i.e., $K=2$, on the performance of algorithm is discussed. A uniform linear array with $M=6$ is used to estimate DOA information of two independent sources in space. The angle of one source $\theta_{1}$ is randomly selected between $\left[-60^{\circ}, 60^{\circ}\right]$, and the other one is $\theta_{1}+\delta^{\circ}$, where $\delta$ is changed from $2^{\circ}$ to $5^{\circ}$ with uniform step $0.5^{\circ}$. One hundred Monte Carlo simulations are carried out for each $\delta$. The number of snapshots $L$ is 200 and SNR $=20 \mathrm{~dB}$. Figure 5 shows the RMSEs of five different algorithms at different angle intervals.

From the simulation results shown in Fig. 5, it is not difficult to see that among the five algorithms mentioned above, $\ell_{1}$-svd algorithm is most affected by the angle interval of the source, and MUSIC and OGSBL are slightly better. Since SPA and the proposed algorithm in this paper do not depend on spatial discrete grids or spatial spectral peak 


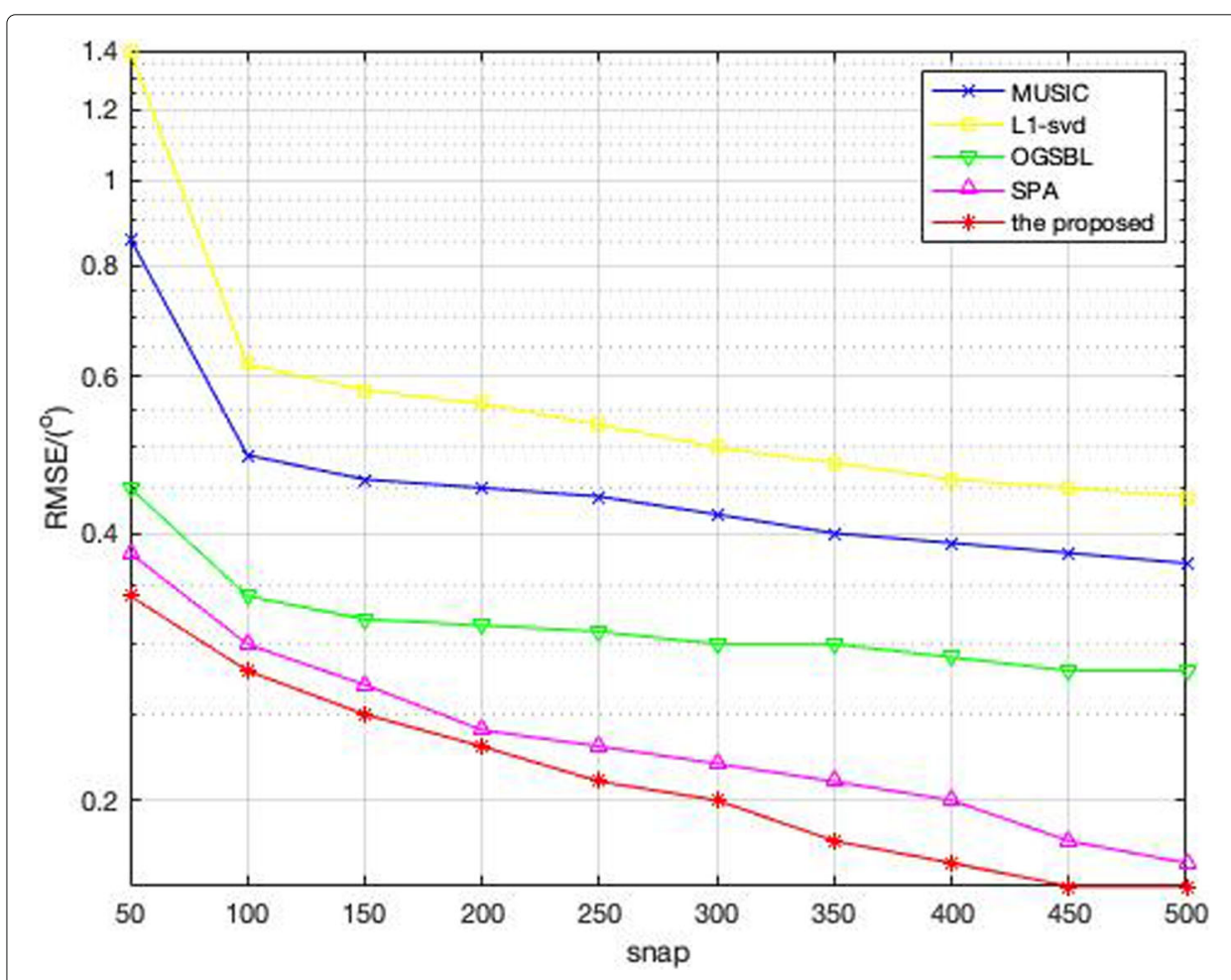

Fig. 4 RMSEs of five algorithms versus snapshots

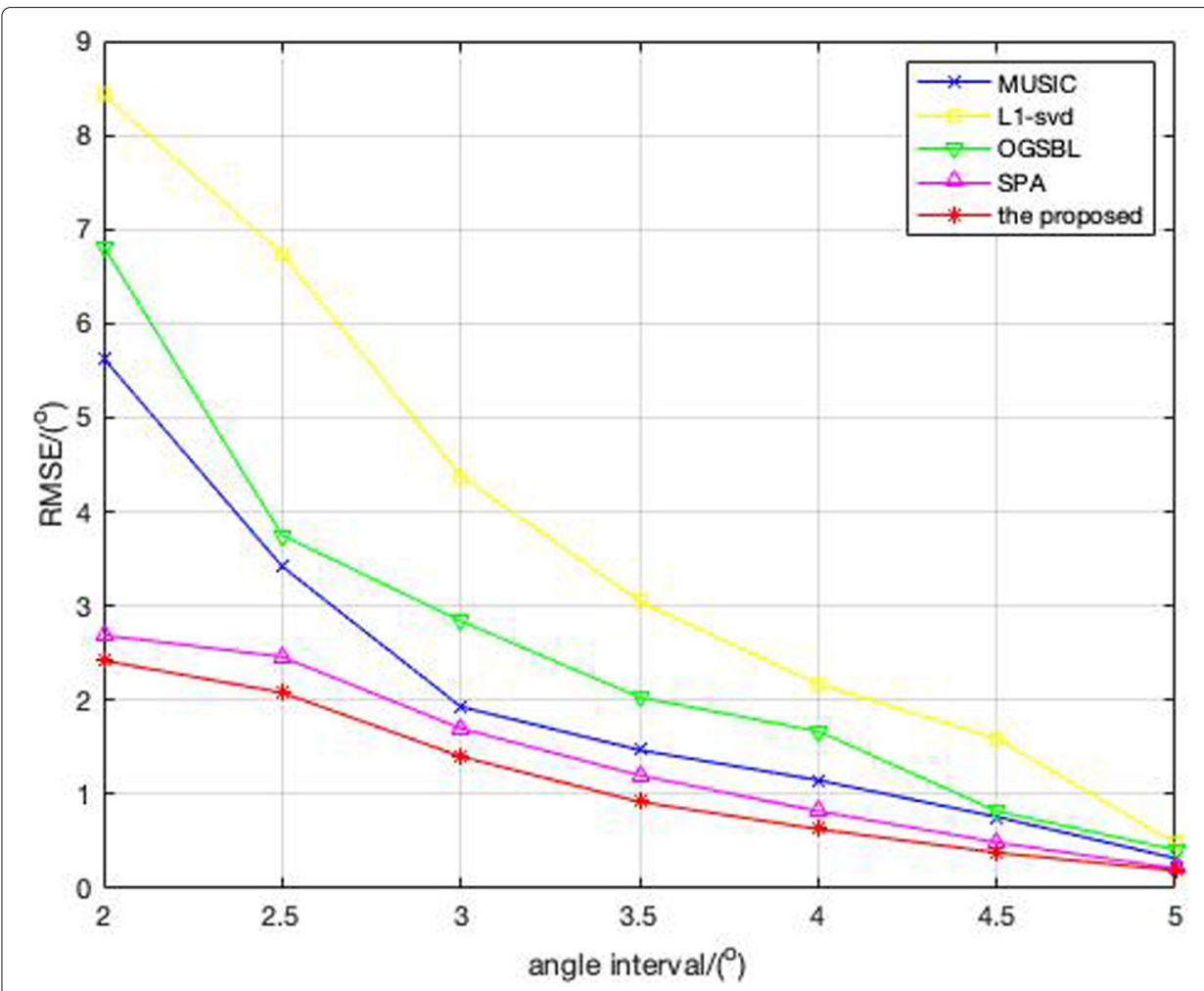

Fig. 5 RMSEs of five algorithms versus angle intervals 
search, outstanding estimation performance can be obtained when the source location is very close, which just proves the superiority of gridless DOA estimation algorithm.

\subsection{Run time comparisons}

At last, we compare the run time of five algorithms. From the analysis of the computational complexity in Section 3.3.3, we can find that the running time of MUSIC, $\ell_{1}$-svd, and OGSBL algorithm is closely related to the number of spatial discrete grid points $N$. However, the parameters that play a decisive role in the running time of SPA and the proposed algorithm only include the number of elements $M$ and snapshots $L$. At the same time, it is not difficult to see that $M$ has a far greater impact on the complexity than $L$. Therefore, in the following experiment, we will compare the run time of the above five algorithms under different $M$.

The number of sources is $K=2$, and the method of selecting DOA parameters $\theta_{k}$ is the same as that in the experiment corresponding to Fig. 3. The number of snapshots is $L=200$. In order to compare the running time of the algorithm under different values of $N$, the number of spatial discrete grid points is 180 and 360, respectively, i.e., $N=180$ and $N=360$. Figure 6 shows the running time curves of the above five algorithms under different $M$.

From the simulation results shown in Fig. 6, it can be seen that the running time of the five algorithms increases with the number of elements $M$. MUSIC algorithm needs the shortest running time because it does not involve the iterative optimization process. When the number of grid points is $N=180$, the running time of $\ell_{1}$-svd and OGSBL algorithm is less than that of gridless DOA estimation algorithms. However, when $N$ becomes larger, the running time of these algorithms increases greatly, and when $N=360$, it has gone beyond the gridless algorithms. In addition, it is worth mentioning that the running time of the proposed algorithm is always less than that of SPA when $M$ is the same, which just verifies the conclusion of Section 3.3.1.

\section{Results and discussion}

In this paper, inspired by the SPA and FRI signal reconstruction theory, a modified algorithm is proposed to improve the accuracy of DOA estimation by overcoming the

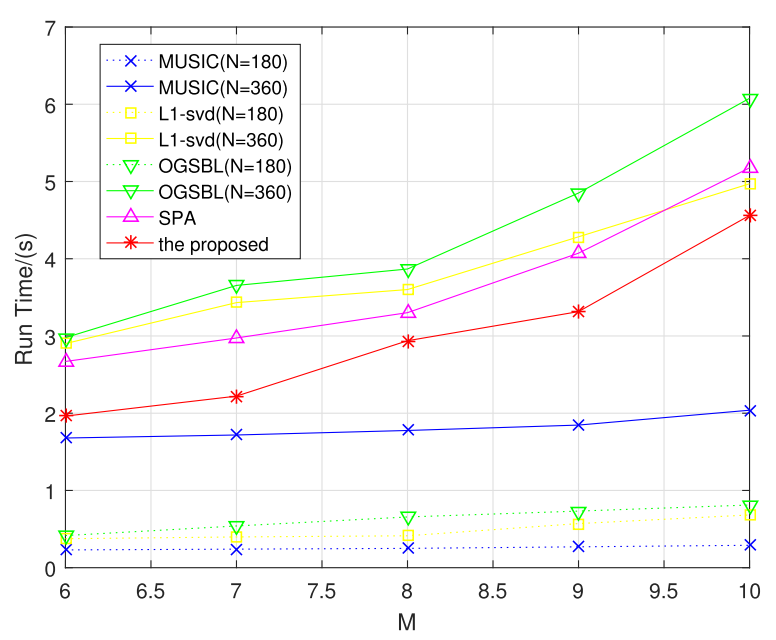

Fig. 6 Run time of five algorithms versus $M$ 
problem of grid mismatch, which constantly arises in sparsity-based DOA estimation algorithms. The proposed algorithm takes advantage of the covariance fitting criterion to reduce the impact of Gaussian white noise limited number of snapshots, rather than directly using the received signals or covariance matrix of antenna sensor array. Then, the annihilation filter equation is constructed by taking advantage of the off-diagonal elements in the optimized covariance matrix. Finally, the DOAs of the sources can be retrieved from the zeros of annihilation equation. The proposed algorithm not only does not depend on spatial discrete grids, but also considers the influence of Gaussian white noise on the reconstruction model and makes full use of the observed data of multi-snapshots.

\section{Abbreviations}

DOA: Direction of arrival; FRI: Finite rate of innovation; ULA: Uniform linear array; MMV: Multiple measurement vector; SNR: Signal-to-noise ratio; ANM: Atomic norm minimization; SMV: Single measurement vector; SPA: Sparse and parametric approach; SDP: Semidefinite programming; RMSE: Root mean square error

\section{Acknowledgements}

Not applicable.

\section{Authors' contributions}

The idea of this work was proposed by Tao Chen. Lin Shi performed the experiments and analyzed the simulation results. Yongzhi Yu wrote the paper. The author(s) read and approved the final manuscript.

\section{Funding}

This work was supported in part by the National Natural Science Foundation of China under Grant 62071137 and in part by the Fundamental Research Funds for the Central Universities.

\section{Availability of data and materials}

All data generated or analyzed during this study are included in this published article.

\section{Consent for publication}

Our manuscript does not contain any individual person's data in any form.

\section{Competing interests}

The authors declare that they have no competing interests.

Received: 17 July 2019 Accepted: 30 September 2020

Published online: 28 October 2020

References

1. M. Guo, Y. D. Zhang, T. Chen, DOA estimation using compressed sparse array. IEEE Trans. Signal Process. 66, 4133-4146 (2018)

2. T. Chen, L. Shi, L. Guo, Sparse DOA estimation algorithm based on fourth-order cumulants vector exploiting restricted non-uniform linear array. IEEE Access. 7, 9980-9988 (2019)

3. R. Schmidt, Multiple emitter location and signal parameter estimation. IEEE Trans. Signal Process. 34, $276-280$ (1986)

4. R. Roy, T. Kailath, ESPRIT-estimation of signal parameters via rotational invariance techniques. IEEE Trans. Acoust. Speech Signal Process. 37, 984-995 (1989)

5. D. Donoho, Compressed sensing. IEEE Trans. Inf. Theory. 52, 1289-1306 (2006)

6. X. Wei, Y. Yuan, Q. Ling, DOA estimation using a greedy block coordinate descent algorithm. IEEE Trans. Signal Process. 60, 6382-6394 (2012)

7. M. Hyder, K. Mahata, Direction-of-arrival estimation using a mixed $\ell_{2.0}$ norm approximation. IEEE Trans. Signal Process. 58, 4646-4655 (2010)

8. D. Malioutov, M. Cetin, A. Willsky, A sparse signal reconstruction perspective for source localization with sensor arrays. IEEE Trans. Signal Process. 53, 3010-3022 (2005)

9. Z. Yang, L. Xie, C. Zhang, Off-grid direction of arrival estimation using sparse Bayesian inference. IEEE Trans. Signal Process. 61, 38-43 (2012)

10. T. Chen, H. Wu, L. Guo, L. Liu, A modified rife algorithm for off-grid DOA estimation based on sparse representations. 15, 29721-29733 (2015)

11. G. Tang, B. Bhaskar, P. Shah, B. Recht, Compressed sensing off the grid. IEEE Trans. Inf. Theory. 59, 7465-7490 (2013)

12. Z. Yang, L. Xie, On gridless sparse methods for line spectral estimation from complete and incomplete data. IEEE Trans. Signal Process. 63, 3139-3153 (2015)

13. B. Bhaskar, G. Tang, B. Recht, Atomic norm denoising with applications to line spectral estimation. IEEE Trans. Signal Process. 61, 5987-5999 (2013)

14. Y. Li, Y. Chi, Off-the-grid line spectrum denoising and estimation with multiple measurement vectors. IEEE Trans. Signal Process. 64, 1257-1269 (2016) 
15. P. Stoica, P. Babu, J. Li, SPICE: a sparse covariance-based estimation method for array processing. IEEE Trans. Signal Process. 59, 629-638 (2011)

16. P. Stoica, P. Babu, SPICE and LIKES: two hyperparameter-free methods for sparse-parameter estimation. Signal Process. 92, 1580-1590 (2012)

17. M. Vetterli, P. Marziliano, T. Blu, Sampling signals with finite rate of innovation. IEEE Trans. Signal Process. 50, 1417-1428 (2002)

18. V. Chandrasekaran, B. Recht, P. A. Parrilo, A. S. Willsky, The convex geometry of linear inverse problems. Found. Comput. Math. 12, 805-849 (2012)

19. Z. Yang, L. Xie, C. Zhang, A discretization-free sparse and parametric approach for linear array signal processing. IEEE Trans. Signal Process. 62, 4959-4973 (2014)

20. H. Pan, T. Blu, M. Vetterli, Towards generalized FRI sampling with an application to source resolution in radioastronomy. IEEE Trans. Signal Process. 65, 821-835 (2017)

21. H. Pan, R. Scheibler, E. Bezzam, I. Dokmanić, M. Vetterli, in 2017 IEEE International Conference on Acoustics, Speech and Signal Processing (ICASSP), FRIDA: FRI-based DOA estimation for arbitrary array layouts, (New Orleans, 2017), pp. 3186-3190. https://doi.org/10.1109//CASSP.2017.7952744

22. Y. Pan, G. Luo, H. Jin, X. Zhang, C. Yin, DOA estimation with planar array via spatial finite rate of innovation reconstruction. Signal Process. 153, 47-57 (2018)

23. T. Blu, P. L. Dragotti, M. Vetterli, P. Marziliano, L. Coulot, Sparse sampling of signal innovations. IEEE Signal Process. Mag. 25, 31-40 (2008)

24. S. Boyd, L. Vandenberghe, Convex Optimization. (Cambridge Univ., Cambridge, 2004)

25. CVX: MATLAB software for disciplined convex programming (2008). http://cvxr.com/cvx

\section{Publisher's Note}

Springer Nature remains neutral with regard to jurisdictional claims in published maps and institutional affiliations.

\section{Submit your manuscript to a SpringerOpen ${ }^{\circ}$} journal and benefit from:

- Convenient online submission

- Rigorous peer review

- Open access: articles freely available online

High visibility within the field

- Retaining the copyright to your article

Submit your next manuscript at $\gg$ springeropen.com 